\title{
Prevention and management of complications following tracheal resections-lessons learned at the Massachusetts General Hospital
}

\author{
Luis F. Tapias ${ }^{1,2}$, Douglas J. Mathisen ${ }^{1,2}$ \\ ${ }^{1}$ Division of Thoracic Surgery, Massachusetts General Hospital, Boston, MA, USA; ${ }^{2}$ Harvard Medical School, Boston, MA, USA \\ Correspondence to: Douglas J. Mathisen, MD. Division of Thoracic Surgery, Massachusetts General Hospital, 55 Fruit Street, Founders 7, Boston, MA \\ 02114, USA. Email: dmathisen@mgh.harvard.edu.
}

\begin{abstract}
Tracheal surgery has developed and evolved over the last 50 years, becoming an integral part of the clinical practice of modern thoracic surgeons. Today, surgeons can safely and effectively operate on most patients suffering from post-intubation tracheal stenosis, tracheal tumors and other pathologies that result in an abnormal shape and function of the central airways. The Massachusetts General Hospital (MGH) in Boston witnessed first-hand the birth and growth of tracheal surgery under Dr. Hermes Grillo, becoming a reference center and accumulating one of the largest institutional experiences in the world. This vast experience of the management of patients with tracheal pathologies has allowed our group at MGH to refine the care of these patients, including their judicious preoperative evaluation, meticulous surgical treatment, and dedicated postoperative care. However, this experience has also provided valuable lessons on the potential complications that can arise after tracheal surgery. Here, we attempt to accumulate our institutional knowledge and experience to provide a summary of the key aspects to prevent complications following tracheal resections. Unfortunately, complications can occur despite strict adherence to the core principles of tracheal surgery, and therefore, we also highlight our institutional experience with the early recognition and management of the most common and dreaded complications.
\end{abstract}

Keywords: Tracheal stenosis; tracheal tumor; trachea; thoracic surgery; airway management; anastomosis

Submitted Nov 17, 2017. Accepted for publication Jan 08, 2018.

doi: $10.21037 /$ acs.2018.01.20

View this article at: http://dx.doi.org/10.21037/acs.2018.01.20

\section{Introduction}

Segmental resection and reconstruction of the central airway can be performed successfully and safely in the majority of patients. The surgical techniques for tracheal surgery have been refined since their initial description in the mid- $20^{\text {th }}$ century. Patients with surgical problems of the central airways are mainly treated at specialized highvolume centers where they receive multidisciplinary care from thoracic surgery, anesthesiology, otolaryngology and critical care. In any case, tracheal and laryngotracheal resection and reconstruction should be part of the armamentarium of the thoracic surgeon. Here, we review the core principles of tracheal surgery and prevention, recognition and management of potential complications. Adherence to these principles will maximize the chances of a successful operation and decrease the chances of postoperative complications. Complications after tracheal resections are infrequent but can be life-threatening. Prevention, anticipation and early recognition are keys to effectively manage complications.

\section{Indications and contraindications}

\section{Indications}

Tracheal resection with primary reconstruction is most frequently performed for the treatment of post-intubation tracheal injuries (1). The use of endotracheal tubes with low-pressure cuffs, careful intubation avoiding unnecessary trauma, meticulous technique when performing a tracheostomy and judicious postoperative care can avoid the development of tracheal stenosis as a complication. However, recent observations suggest that as percutaneous 
tracheostomy techniques gain popularity, these may become the most frequent cause of tracheal stenosis, with added complexity as involvement of the cricoid cartilage is frequentresulting in laryngotracheal stenosis (2). Other indications for tracheal resection and reconstruction include tracheal tumors of both primary (e.g., squamous cell carcinoma, adenoid cystic carcinoma) and metastatic origins (e.g., bronchogenic carcinoma, thyroid carcinomas) (3). Idiopathic laryngotracheal stenosis is rare but seen with increased frequency, affecting almost exclusively young females (4). Other patients that may undergo tracheal resection and reconstruction are those with tracheoesophageal fistula (TEF) (5), tracheoinnominate fistula (TIF), post-infectious lesions or congenital problems (6).

\section{Contraindications}

Absolute contraindications are related to poor surgical candidacy due to significant medical comorbidities or lack of sufficient healthy trachea precluding primary anastomosis secondary to extension or local invasion of a tracheal tumor or previous tracheal surgery.

\section{Special considerations and high-risk patients}

There are a few important special situations in airway surgery that deserve special attention. The need for prolonged postoperative mechanical ventilation should be anticipated. Positive-pressure ventilation can put stress on the airway anastomosis and increase the risk of complications. The anesthetic plan should include every effort to permit routine extubation at the end of the procedure. The chronic use of corticosteroids can lead to impairment of airway anastomotic healing. Ideally, patients should be off steroids for 2-4 weeks before surgery. Preoperative steroids do not prevent airway edema and should not be used to that end. Also, prior radiation therapy has the potential to impact wound healing. In highly selected patients, tracheal resection and reconstruction can still be performed (7). In these cases, buttressing of the anastomosis with well-vascularized pedicled tissue should be strongly considered. Radiation therapy should be avoided preoperatively in patients with resectable tracheal tumors. Finally, diabetic patients are at an increased risk of developing anastomotic complications (8), likely due to impaired microvasculature affective healing at the anastomosis. Improved glycemic control before surgery may be beneficial on these patients.

Previous surgery can present challenges. Revisional tracheal surgery poses a particular challenge, as excessive anastomotic tension is frequent due to fibrosis limiting mobilization of tissues and a resulting shorter trachea (9). The presence of a tracheostomy before surgery suggests a traumatized airway at baseline more susceptible to bacterial colonization, inflammation, impaired wound healing and malacic segments.

Patients with tracheal stenosis related to granulomatosis with polyangiitis (i.e., Wegener's granulomatosis) are poor candidates for resection as the disease course is unpredictable and relapses are frequent. Bronchoscopic treatments are considered first line in these patients. Highly selected patients with refractory airway stenosis may benefit from resection, although they may require subsequent bronchoscopic interventions (10).

Pediatric patients do not tolerate tension on the airway anastomosis as well as adults. Therefore, the length of trachea that can be resected is proportionally lower than in adult patients (6).

\section{Surgical principles}

There are some core principles that apply to airway surgery that need to be followed strictly to maximize the chances of a successful operation and to minimize the risk of postoperative complications. The surgeon ought to be aware and exercise great judgement in knowing the limits of airway resection, the acceptable extension of dissection with subsequent interruption of blood supply, and the acceptable amounts of anastomotic tension.

(I) Length of resection: adult patients can have up to $50 \%$ of the trachea resected. However, the incidence of postoperative anastomotic complications increases significantly when resections exceed $4 \mathrm{~cm}$ in length (8). In pediatric patients, complications increase when more than $30 \%$ of the trachea is resected (6);

(II) Tumor resectability: preoperative imaging studies and bronchoscopic assessment are of the upmost importance. It will help determining the extension of the diseased segment and the quality of the tissues. However, it may not reliably indicate the degree of involvement of neighboring structures, therefore, the final decision to perform a tracheal resection is made in the operating room. The resectability of the tumor needs to be determined before any structures are divided. Liberal use of frozen-section analysis of margins will help tailor the approach. Microscopic positive margins of 
resection can be accepted in cases of adenoid cystic carcinoma given its tendency to spread along the submucosal plane $(11,12)$. Further details on the surgical treatment of tracheal tumors is detailed elsewhere in this issue;

(III) Airway dissection: careful dissection of the airway should be performed to preserve the blood supply to the trachea to avoid ischemia at the anastomosis. Circumferential dissection is only performed at the levels of the pathology. Lateral dissection proximal and distal to the proposed lines of transection should be limited to approximately $1 \mathrm{~cm}$ to avoid devascularization;

(IV) Anastomotic tension: this is the largest contributor to anastomotic complications. Careful attention needs to be paid to the length of the airway segment to be resected, as tension and the incidence of anastomotic complications increases with the length of resection. Release maneuvers are helpful in reducing anastomotic tension;

(V) Release maneuvers: multiple release maneuvers exist to help reduce tension at the tracheal anastomosis (13). Any surgeon performing tracheal or laryngotracheal resections and reconstructions should be familiar with these techniques. Details on these maneuvers are provided elsewhere in this issue. Briefly, neck flexion is the simplest maneuver and allows the caudal motion of the proximal trachea. The suprahyoid release is helpful in cases of laryngotracheal or proximal tracheal resection. Mobilization of the pretracheal plane provides additional mobility. Blunt dissection of this plane using mediastinoscopy facilitates dissection in cases of distal tracheal resection that are approached via thoracotomy. If indicated, mediastinoscopy should be performed at the time of proposed resection to avoid scar formation. The division of the inferior pulmonary ligament should be performed routinely in cases of distal tracheal resection that are approached via thoracotomy. Finally, the inferior hilar release reduces anastomotic tension significantly. This release is best accomplished before starting airway resection by making a U-shaped incision in the pericardium below the inferior pulmonary vein, with intrapericardial division of the raphe extending between the inferior pulmonary vein and the inferior vena cava. Additional length may be gained by completely incising the pericardium around the hilar vessels, which would be denominated as a complete hilar release;

(VI) Anastomotic technique: the preferred technique for airway anastomosis at the authors' institution is the sequential placement of multiple lubricated interrupted sutures at $2-3 \mathrm{~mm}$ intervals using absorbable material, such as 4-0 polyglactin 910 $\left(\right.$ Vycril $^{\circledR}$, Ethicon, Somerville, NJ, USA). Full thickness traction sutures are placed laterally on both sides of the trachea to aid decreasing tension. The use of absorbable suture material is preferred to prevent granuloma formation or stenosis at the anastomosis (14). The knots are tied outside the airway lumen after all sutures have been placed;

(VII) Surveillance bronchoscopy: routine early bronchoscopic evaluation of the anastomosis ( 1 week) is the key to recognizing anastomotic complications, as ischemia leading to mucosal abnormalities can be detected sooner than when anastomotic complications present with symptoms or a wound infection.

\section{Complications of tracheal resections}

Even after careful patient selection and judicious surgical technique, complications can arise in the postoperative period. Tracheal resections and reconstructions have been associated with a mortality rate of $0 \%$ to $5 \%$ and an overall morbidity rate of $9 \%$ to $45 \%(2,8,15-22)$ (Table 1$)$.

Procedure-specific complications after tracheal resections are mostly related to the airway anastomosis and include the development of granulomas at the anastomosis, anastomotic separation, and benign anastomotic strictures or recurrent stenosis. Additionally, patients are at risk of fistula formation (e.g., tracheoinnominate, tracheoesophageal), laryngeal problems [including glottic edema and recurrent laryngeal nerve (RLN) injury], and swallowing dysfunction.

At our institution, adherence to the previously mentioned surgical principles has yielded excellent results. The largest series reporting postoperative outcomes after tracheal resection and reconstruction comes from the experience at the Massachusetts General Hospital (MGH) (8). This retrospective study reviewed 901 patients who underwent tracheal and laryngotracheal resection and reconstruction over a 28 -year period. The most frequent indications for surgery were post-intubation tracheal stenosis (589 patients, $65.4 \%)$ and tumors involving the trachea $(208,23.1 \%)$, followed by patients with idiopathic laryngotracheal stenosis 


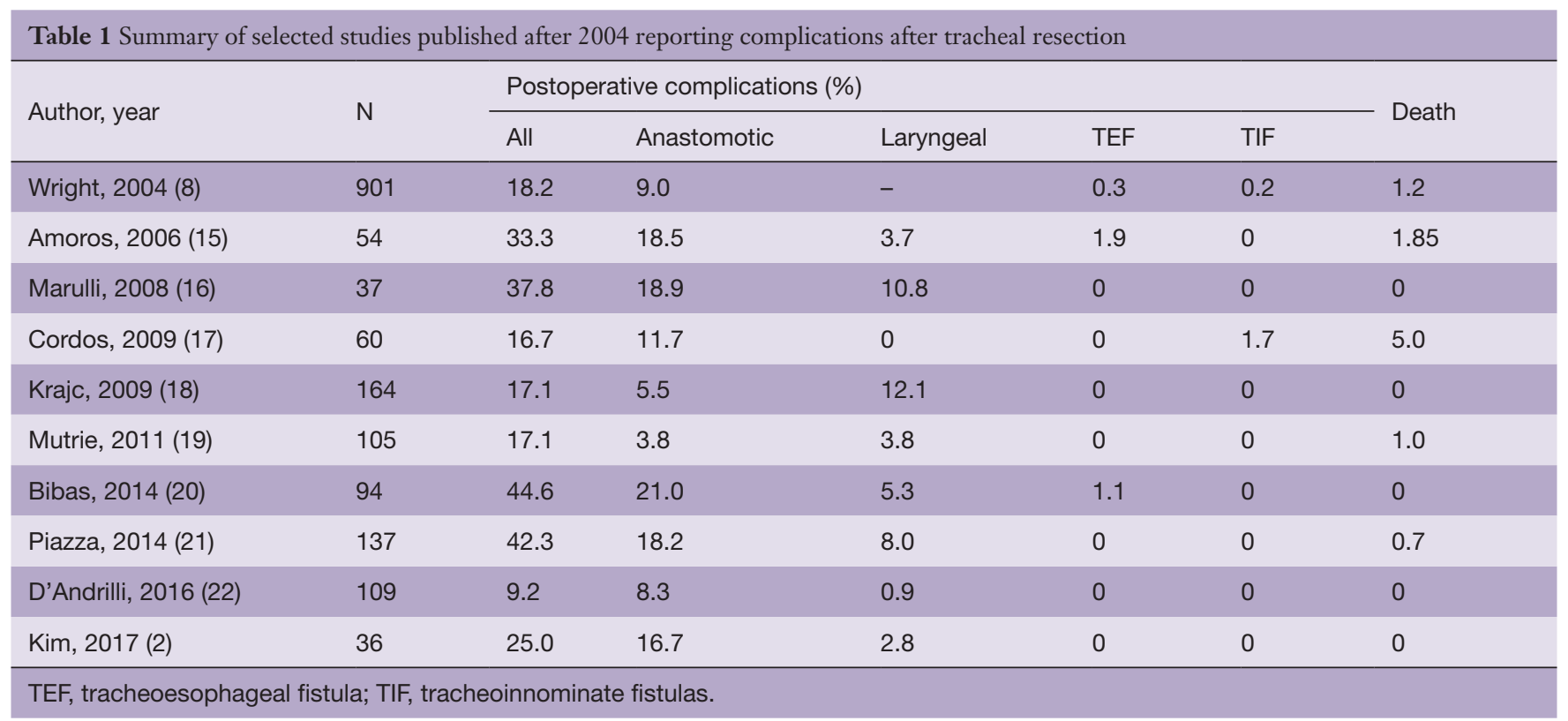

(83, 9.2\%) and TEF (21, 2.3\%). This study revealed a postoperative mortality rate of $1.2 \%$ and an overall complication rate of $18.2 \%$, with anastomotic complications occurring in $9 \%$ of patients (8). The results from our institutional experience are considered the benchmark in the field and they continue to compare favorably to more recently published series (Table 1).

Importantly, the MGH study provided a robust statistical analysis on a large cohort of patients that identified several risk factors for the development of anastomotic complications. These factors should be considered carefully when evaluating patients for potential tracheal resection and reconstruction. Preoperative factors include the presence of diabetes and redo procedures, each independently increases the risk of anastomotic complications by a factor of three. Pediatric patients (i.e., 17 years old or less) have more than twice the risk of developing anastomotic complications when compared to older patients. Having a tracheostomy before surgery increased the risk of postoperative anastomotic complications by $\sim 80 \%$. Intraoperatively, patients that required a resection exceeding $4 \mathrm{~cm}$ in length had a 2 -fold increase in anastomotic complications, whereas, patients that required a laryngotracheal resection carried an $80 \%$ higher risk of developing complications. Avoiding postoperative anastomotic complications is important as it impacts mortality. Patients who suffered anastomotic complications experienced a postoperative mortality rate of $7.4 \%$ compared to $0.6 \%$ in patients without anastomotic complications $(\mathrm{P}=0.0001)$ (8).

\section{Anastomotic complications}

\section{Granulation tissue}

Some degree of granulation tissue is expected postoperatively, but this rarely results in airway obstruction. Clinically significant granulation tissue causing airway obstruction is now infrequently encountered with the routine use of absorbable suture material to construct the airway anastomosis (7 of 901 patients in the MGH experience, or $0.7 \%$ ) (8). Patients may present with stridor days to weeks after surgery. Prompt evaluation with flexible bronchoscopy is indicated in these cases to assess the problem. Granulation tissue at the suture line can be debrided during bronchoscopy, and for most patients this is all that is needed. Local injection of triamcinolone has been reported to prevent reformation of granulations (23). A T-tube can stabilize the airway when granulation tissue formation is severe and recurrent airway obstruction is a concern. Finally, reoperation can be considered for severe refractory cases, but in our experience this is seldom required.

\section{Anastomotic separation}

Separation of the anastomosis is a devastating complication. 
In the MGH experience, this occurred infrequently (37 of 901 patients, or $4.1 \%$ ) (8). The presentation is early within days to the first 2 weeks after surgery. Patients may present with stridor, cough, increased secretions, hemoptysis, subcutaneous emphysema or a wound infection. Complete loss of the airway is rare but carries a high fatality rate. For the patient presenting in extremis, the priority is stabilizing the airway. Concomitant laryngeal edema may make orotracheal intubation difficult. Reopening the incision at the bedside to cannulate the distal airway is a measure of last resort. If the patient is stable, a CT scan of the neck and chest can help assessing for fluid collections in the neck or mediastinum and signs of pneumonia.

If separation is suspected, the patient must be taken immediately to the operating room for evaluation and stabilization of the airway. The defect is usually located anteriorly as this is the site with the highest tension. Small separations can be watched, especially if the area is buttressed with a vascularized tissue flap. Antibiotic therapy, control of contamination by drainage, voice rest and neck flexion are instituted to allow healing of the anastomosis. Hyperbaric oxygen therapy may help promoting and expediting healing (24). In cases with anastomotic separations larger than several millimeters the neck should be explored. These cases should usually be managed with an airway appliance. A T-tube can be used if the upper airway is patent, however, if there is significant laryngeal edema a tracheostomy should be placed through the defect. Immediate revision of the anastomosis should be avoided, although it can be considered in select cases caused by an episode of violent coughing or neck hyperextension in the early postoperative period.

\section{Benign strictures or restenosis}

Benign strictures or restenosis have an insidious onset and are the result of anastomotic problems including tension, ischemia, and/or subclinical separation leading to scar formation. In the MGH experience, this occurred infrequently (37 of 901 patients, or $4.1 \%$ ) (8). When proceeding with a tracheal resection for post-intubation stenosis, all of the trachea involved by severe inflammation must be removed to prevent recurrence. Patients may present months after surgery with symptoms of airway obstruction. The diagnosis is confirmed with flexible bronchoscopy.

Management consists of dilation of the stricture using rigid bronchoscopy or balloon dilation. If dilations do not provide durable relief and are required more often than every
3-6 months, then reoperation should be considered. However, re-intervention should be deferred for 6-12 months to allow for complete resolution of healing after the index surgery. Silicone stents or a T-tube may be required to temporize patients prior to re-resection or as a long-term solution to stabilize the airway that is not amenable to re-resection.

\section{Fistulas}

\section{TIF}

TIFs are rare but almost invariably carry a very poor prognosis. These patients typically manifest with massive hemoptysis and severe acute respiratory and hemodynamic compromise and may die precipitously. This is a very rare complication, encountered in 2 of $901(0.2 \%)$ cases in the $\mathrm{MGH}$ experience (8), and in only one case among 796 patients $(0.1 \%)$ reported in other series shown in Table 1. TIF results from anterior separation causing infection and inflammation that erodes into the innominate artery. The massive bleed is often preceded by a "sentinel bleed". Small volume hemoptysis in a stable patient may be investigated with CT angiography prior to bronchoscopic evaluation. However, significant hemoptysis should prompt emergent evaluation and intervention (25). Emergent placement of a cuffed endotracheal tube can potentially seal the bleeding site while operative plans are set in motion. The surgical approach involves a combination of a collar incision and a median sternotomy. The innominate artery is controlled proximally and distally and the segment involved in the fistula is resected. The cut edges are oversewed and covered with healthy tissue. Neurologic complications are rare in the presence of a previously normal circulation. If available, intraoperative electroencephalogram monitoring can be used while the artery is clamped to detect any changes, in which case reconstruction with an autologous saphenous vein graft is recommended given the contaminated nature of the field. The defect on the tracheal anastomosis should be repaired and buttressed at the same time. Details are provided elsewhere in this issue.

TIFs can be prevented by limiting dissection behind the innominate artery and by staying close to the trachea. Healthy, vascularized tissue should be interposed between the trachea and the innominate artery whenever the anastomotic suture line is close to the artery.

\section{TEF}

TEFs are rare complications after tracheal resection 
and reconstruction. In the MGH series, only 3 of 901 patients $(0.3 \%)$ suffered this complication. Other series have reported rates of $0 \%$ to $1.9 \%$ (Table 1). It results from separation of the posterior membranous wall of the anastomosis or from esophageal injury during the initial operation. Patients may present in a delayed fashion with cough, dysphagia and aspiration pneumonia. The diagnosis is confirmed with an esophagram, esophagoscopy and bronchoscopy. TEFs should be repaired when encountered. Inflammation and infection need to clear before attempting repair and broad-spectrum antibiotics and drainage of neck or mediastinal collections is indicated. The airway can be temporized with a T-tube or a cuffed tracheostomy. A venting gastrostomy can be useful to prevent reflux with subsequent aspiration, and a jejunostomy to provide nutritional support. Repair of the fistula can be accomplished by simple division and closure or by segmental tracheal resection with repair of the esophagus. Details are provided elsewhere in this issue.

\section{Laryngeal complications}

\section{Laryngeal edema}

This is a more frequent problem in cases of laryngotracheal resection (4). Patients present with hoarseness and on occasion with stridor. Also, this can be detected early on surveillance bronchoscopy. Bronchoscopy is diagnostic and helps ruling out concomitant anastomotic complications. Mild cases are managed with head elevation, voice rest, steroids, diuretics, and nebulized epinephrine. A mixture of helium and oxygen (Heliox) has a lower density than a mixture of nitrogen and oxygen and results in decreased resistance to flow within the airways, which results in decreased work of breathing. More severe cases with impending loss of the airway can be managed with intubation using a small-uncuffed endotracheal tube. Tracheostomy needs to be considered in patients with persistent edema after a few days of intubation.

\section{RLN palsy}

The state and function of the glottis and subglottic area should be established preoperatively. RLN injury following tracheal or laryngotracheal resection and reconstruction is rare if proper technique is used and dissection stays close to the tracheal wall. It was encountered in only $0.8 \%$ of cases in our experience with laryngotracheal resections (4).
It is our practice to avoid exposing the tracheoesophageal groove and to avoid attempts at identifying the course of the nerve. Some degree of postoperative hoarseness is common and may result from laryngeal edema or transient RLN palsy secondary to inflammation or traction. Patients also exhibit a weak cough. Bedside flexible laryngoscopy can help determine the mobility of the vocal cords. However, an immobile cord can recover over time, and therefore, medialization laryngoplasty should not be performed within the first 6 months after surgery. Temporary injection of the cord is indicated if there is objective evidence of aspiration. These patients should be referred to a speech pathologist to work on improving swallowing and phonation. In the rare case with bilateral RLN injury, tracheostomy is indicated.

\section{Swallowing dysfunction}

Significant swallowing dysfunction is not common after tracheal surgery. Multiple factors can contribute to postoperative swallowing dysfunction including the presence of laryngeal edema, RLN palsy, having a suprahyoid release, and extensive tracheal resections. The last two affect the normal mobility of the larynx affecting the swallowing mechanism. Older age is another risk factor. Patients need to be kept nil per os during the early postoperative period to avoid catastrophic aspiration events. Improvement is expected in most patients over time. Formal evaluation by a speech pathologist is mandatory before advancing diet on these patients.

\section{Conclusions}

Careful patient selection and attention to technical details should minimize postoperative complications following tracheal resection. Complications when they do occur are often serious and require early detection and aggressive treatment. Unsuccessful management of complications often leads to death.

\section{Acknowledgements}

None.

\section{Footnote}

Conflicts of Interest: The authors have no conflicts of interest to declare. 


\section{References}

1. Cooper JD, Grillo HC. The evolution of tracheal injury due to ventilatory assistance through cuffed tubes: a pathologic study. Ann Surg 1969;169:334-48.

2. Kim SS, Khalpey Z, Hsu C, et al. Changes in Tracheostomy- and Intubation-Related Tracheal Stenosis: Implications for Surgery. Ann Thorac Surg 2017;104:964-70.

3. Grillo HC, Mathisen DJ. Primary tracheal tumors: treatment and results. Ann Thorac Surg 1990;49:69-77.

4. Wang H, Wright CD, Wain JC, et al. Idiopathic Subglottic Stenosis: Factors Affecting Outcome After Single-Stage Repair. Ann Thorac Surg 2015;100:1804-11.

5. Muniappan A, Wain JC, Wright CD, et al. Surgical treatment of nonmalignant tracheoesophageal fistula: a thirty-five year experience. Ann Thorac Surg 2013;95:1141-6.

6. Wright CD, Graham BB, Grillo HC, et al. Pediatric tracheal surgery. Ann Thorac Surg 2002;74:308-13; discussion 314.

7. Muehrcke DD, Grillo HC, Mathisen DJ. Reconstructive airway operation after irradiation. Ann Thorac Surg 1995;59:14-8.

8. Wright CD, Grillo HC, Wain JC, et al. Anastomotic complications after tracheal resection: prognostic factors and management. J Thorac Cardiovasc Surg 2004;128:731-9.

9. Donahue DM, Grillo HC, Wain JC, et al. Reoperative tracheal resection and reconstruction for unsuccessful repair of postintubation stenosis. J Thorac Cardiovasc Surg 1997;114:934-8; discussion 938-9.

10. Costantino CL, Niles JL, Wright CD, et al. Subglottic Stenosis in Granulomatosis With Polyangiitis: The Role of Laryngotracheal Resection. Ann Thorac Surg 2018;105:249-53.

11. Gaissert HA, Grillo HC, Shadmehr MB, et al. Longterm survival after resection of primary adenoid cystic and squamous cell carcinoma of the trachea and carina. Ann Thorac Surg 2004;78:1889-96; discussion 1896-7.

12. Honings J, Gaissert HA, Weinberg AC, et al. Prognostic value of pathologic characteristics and resection margins in tracheal adenoid cystic carcinoma. Eur J Cardiothorac Surg 2010;37:1438-44.

13. Heitmiller RF. Tracheal release maneuvers. Chest Surg Clin N Am 2003;13:201-10.

14. Hsieh CM, Tomita M, Ayabe H, et al. Influence of suture on bronchial anastomosis in growing puppies. J Thorac
Cardiovasc Surg 1988;95:998-1002.

15. Amorós JM, Ramos R, Villalonga R, et al. Tracheal and cricotracheal resection for laryngotracheal stenosis: experience in 54 consecutive cases. Eur J Cardiothorac Surg 2006;29:35-9.

16. Marulli G, Rizzardi G, Bortolotti L, et al. Single-staged laryngotracheal resection and reconstruction for benign strictures in adults. Interact Cardiovasc Thorac Surg 2008; 7:227-30; discussion 230.

17. Cordos I, Bolca C, Paleru C, et al. Sixty tracheal resections--single center experience. Interact Cardiovasc Thorac Surg 2009;8:62-5; discussion 65.

18. Krajc T, Janik M, Benej R, et al. Urgent segmental resection as the primary strategy in management of benign tracheal stenosis. A single center experience in 164 consecutive cases. Interact Cardiovasc Thorac Surg 2009;9:983-9.

19. Mutrie CJ, Eldaif SM, Rutledge CW, et al. Cervical tracheal resection: New lessons learned. Ann Thorac Surg 2011;91:1101-6.

20. Bibas BJ, Terra RM, Oliveira Junior AL, et al. Predictors for postoperative complications after tracheal resection. Ann Thorac Surg 2014;98:277-82.

21. Piazza C, Del Bon F, Paderno A, et al. Complications after tracheal and cricotracheal resection and anastomosis for inflammatory and neoplastic stenoses. Ann Otol Rhinol Laryngol 2014;123:798-804.

22. D'Andrilli A, Maurizi G, Andreetti C, et al. Longterm results of laryngotracheal resection for benign stenosis from a series of 109 consecutive patients. Eur J Cardiothorac Surg 2016;50:105-9.

23. Lanuti M, Mathisen DJ. Management of complications of tracheal surgery. Chest Surg Clin N Am 2003;13:385-97.

24. Stock C, Gukasyan N, Muniappan A, et al. Hyperbaric oxygen therapy for the treatment of anastomotic complications after tracheal resection and reconstruction. J Thorac Cardiovasc Surg 2014;147:1030-5.

25. Allan JS, Wright CD. Tracheoinnominate fistula: diagnosis and management. Chest Surg Clin N Am 2003;13:331-41.

Cite this article as: Tapias LF, Mathisen DJ. Prevention and management of complications following tracheal resectionslessons learned at the Massachusetts General Hospital. Ann Cardiothorac Surg 2018;7(2):237-243. doi: 10.21037/ acs.2018.01.20 